\title{
Auto Segmentation OF Breast Tumor In B-Mode ULTRASOUND ANALYSIS
}

\author{
Shahriar Mahmud Kabir and Mohammed Imamul Hassan Bhuiyan
}

\begin{abstract}
Segmentation or lesion boundary detection in classification of Benign and Malignant breast tumors from B-Mode ultrasound image analysis is a challenging one. In this study, first a suitable frame is chosen by strain and velocity imaging from a raw radio frequency (RF) echo of clinical cases. The consequent B-Mode ultrasound (US) image is calculated and binarized. Finally, the lesion boundary is well-defined by binary dilation technique using MATLAB.
\end{abstract}

Index Terms - Breast Cancer, Segmentation, B-Mode image etc.

\section{INTRODUCTION}

$\mathrm{B}$ reast cancer is flooding significantly in women both in the developed and the developing world in the last few decades. According to the American Cancer Society in 2018, approximately 878,980 women died from cancer where 266,120 of them are died from breast cancer [1]. In Bangladesh the rate of breast cancer occurrence is estimated to be 22.5 per 100000 females of all ages; In case of Bangladeshi women, aged between 15-44 years, breast cancer has the highest prevalence 19.3 per 100000 compared to any other type of cancer [2].

Breast ultrasound imaging has become an essential tool to detect and classify breast cancer tumor now a day. Ultrasound is particularly helpful in distinguishing between a solid mass and a fluid-filled cyst. A hypoechoic solid nodule could be benign (which is known as Fibroadenoma) or could be malignant cancer tumor [3]. Detection of lesion (cancer tumor) region is much more important for feature extraction. Because, few papers used the lesion boundary features as an effective feature in the breast cancer classification purpose.

Karla Horsch in [4] used several features of a breast ultrasound image such as shape feature that was the depth-to-width ratio (DWR) of the lesion, the margin feature that was the normalized radial gradient (NRG),

This paper was received on 22 April 2019, accepted on 19 November 2019. This work was supported financially by Green University of Bangladesh.

Shahriar Mahmud Kabir is an Assistant Professor, Department of EEE,Green University of Bangladesh.E-mail:kabir@eee.green.edu.bd

Mohammed Imamul Hassan Bhuiyan is the Chairperson, Department of Biomedical Engineering, BUET. E-mail: imamul@eee.buet.ac.bd quantify texture which was the autocorrelation in depth of lesion region (COR) and the posterior acoustic behavior feature that was the minimum side difference (MSD) of lesion boundary; for computer aided diagnostics of breast tumors. In this paper the author determined the potential lesion margins through grayvalue thresholding. In that proposed method the lesions were segmented both manually as well as automatically. However, the accuracy obtained do not differ much between manual and automatic segmentation. In [5] Wei-Chih Shen introduced a computer-aided diagnostic (CAD) system where various shape features such as shape class, orientation class, margin class, lesion boundary class, echo pattern class and posterior acoustic feature class are utilized. A total of 265 clinical case images are analyzed in their experiment where 174 are benign and 91 are malignant. A satisfactory performance is reported on that paper where the author defined the lesion boundary manually with the help of a radiologist. In [6] Sharmin Ara employed empirical mode decomposition (EMD) and digital wavelet transform (DWT) followed by a wrapper to obtain a set of non-redundant features for classifying breast tumours and reporting a high degree of accuracy but lesion boundary is defined manually by a radiologist.

In this paper an automatic lesion boundary detection computer-aided diagnostic (CAD) system is proposed which is tested and compared with the pathological result of clinical cases of an open access database consisting of raw ultrasonic signals of 100 clinical cases. The objective is to detect the lesion boundary region precisely from ultrasonic B-Mode image of breast masses, so that it can be classified whether it is benign or malignant more accurately. At first, B-Mode image is extracted from a raw radio frequency (RF) ultrasound data of a clinical case. Next, B-Mode image is binarized so that the CAD system can detects the lesion boundary region automatically. Here, binary dilation technique is applied on the binary image simulated form the corresponding B-Mode using MATLAB.

This paper is organized as follows. Section II gives a short introduction of B-Mode and Binary imaging. The proposed methodology is explained in Section III. The experimental results are presented in Section IV and the conclusion is in Section V. 


\section{B-MODE AND BINARY IMAGING}

Ultrasound (US) is sound waves with frequencies which are higher than those audible to humans $(>20,000 \mathrm{~Hz})$. Ultrasonic images, also known as sonograms, are made by sending pulses of ultrasound into tissue using a probe. The sound echoes off the tissue; with different tissues reflecting varying degrees of sound. These echoes are recorded and displayed as an image to the operator. Ultrasonography (sonography) uses a probe containing multiple acoustic transducers to send pulses of sound into a material. Whenever a sound wave encounters a material with a different density (acoustical impedance), part of the sound wave is reflected back to the probe and is detected as an echo. The time it takes for the echo to travel back to the probe is measured and used to calculate the depth of the tissue interface causing the echo. The greater the difference between acoustic impedances, the larger the echo is. If the pulse hits gases or solids, the density difference is so great that most of the acoustic energy is reflected and it becomes impossible to see deeper. Many different types of images can be formed using sonographic instruments. The most well-known type is a B-mode (brightness mode) image, which displays the acoustic impedance of a two-dimensional cross-section of tissue. In Bmode ultrasound, a linear array of transducers simultaneously scans a plane through the body that can be viewed as a two-dimensional image on screen [7]. On the other hand, a binary image is a digital image that has only two possible values for each pixel. Typically, the two colors used for a binary image are black and white. The color used for the object(s) in the image is the foreground color while the rest of the image is the background color [8]. An example of BMode and Binary images acquired from a medical ultrasound image are shown in Fig. 1.

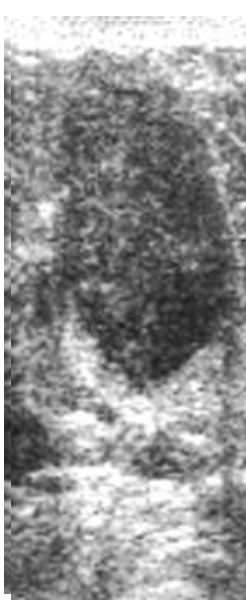

(a)

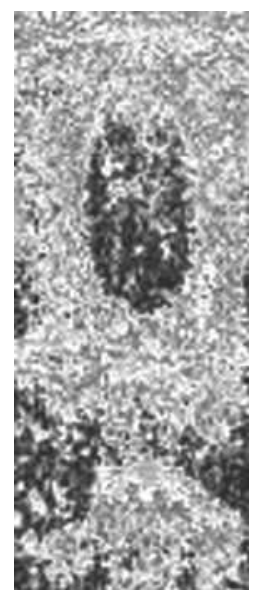

(b)

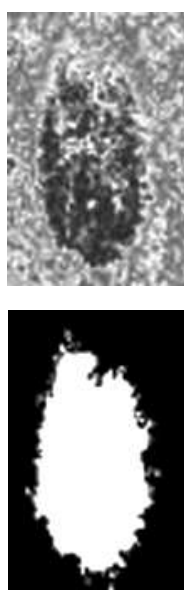

(c)
Fig. 1 Example of B-Mode and binary imaging of a clinical case of benign mass (a) Ultrasound (US) image, (b) Corresponding B-Mode image and (c) Cropped tumor region of B-Mode (upper image) and binary image (lower image).

\section{METHODOLOGY}

The first step in this proposed method is the preprocessing step where we have to choose a suitable frame from an ultrasound raw radio-frequency (RF) ultrasonic echoes (signals) of a patient obtained from an open access database consisting of 100 clinical cases [9]. On that database each clinical case ultrasound images are obtained using an Ultrasonix Sonix-Touch Research ultrasound scanner with an L14-5/38 linear array transducer (centre frequency $10 \mathrm{MHz}$ ). Single focusing beam forming was used with the focusing region always placed at the depth of the lesion. Each scan consisted of $510 \mathrm{RF}$ echo lines. Signals were digitized with $40 \mathrm{MHz}$ sampling frequency. The number of samples in every RF signal depended on the chosen penetration depth. For example, for a $40 \mathrm{MHz}$ sampling rate the distance between adjacent samples is $0.0192 \mathrm{~mm}$ (assuming the speed of sound in tissue is $1540 \mathrm{~m} / \mathrm{s}$ ). Therefore, for a $5 \mathrm{~cm}$ penetration depth 2596 samples were collected, while for a $3 \mathrm{~cm}$ depth the number of samples decreased to 1558. Settings which were used by the, operator had no influence on the characteristics of the raw ultrasonic echoes. The ultrasonic RF echoes were recorded in the Department of Ultrasound, Institute of Fundamental Technological Research Polish Academy of Sciences, from 100 breast focal lesions of patients of the Oncology Institute in Warsaw. 78 women, aged from 24 to 75 years (mean age of 49.5 years) participated in this study. The data were collected between 11/2013 and 10/2015 and all patients provided informed consent before the examination. The study protocol was approved by The Institutional Review Board, Poland. For each clinical $\mathrm{RF}$ data, the strain image and the velocity image are examined using MATLAB function 'DSPView' [10], [11] to obtain the desire frame from which the B-Mode image is calculated. Fig. 2 shows an example of best frame selection by strain and velocity imaging obtained from a raw radio frequency echo of a clinical case. In B-Mode calculation, at first Hilbert transform is applied on the RF frame which is converted into 8-bit unsigned integers. Next, the B-Mode image is subjected to binarization using MATLAB function 'imbinarize' (e.g. Fig. 4b). After that, binary dilation technique is applied to detect boundary region of lesion automatically using MATLAB functions 'bwboundaries' and 'visboundaries' [12], [13].

\section{EXPERIMENTAL RESULTS}

For each ultrasound image lesion scan, the specific individual region of interest (ROI) (i.e. lesion boundary region) was indicated by a radiologist (e.g. Fig. 3). The database consists of 100 lesions, 52 were malignant and 48 were benign. In the group of malignant lesions, all were histologically assessed by core needle biopsy. 37 benign lesions were also histologically assessed. According to current medical standards, the remaining 


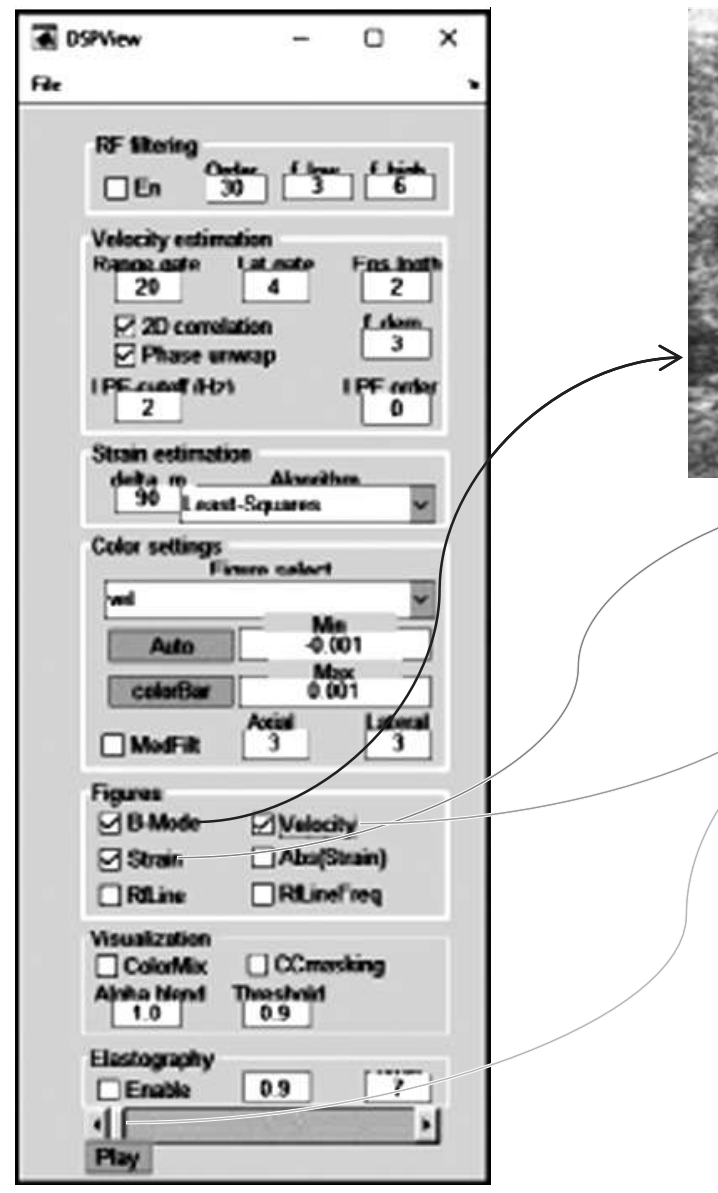

(a)

Fig. 2 Example of best frame selection using (a) MATLAB function 'DSPView' to analyse strain and velocity images of a clinical case (b) Calculated B-Mode image of the corresponding frame (c) Corresponding strain image and (d) Corresponding velocity image.

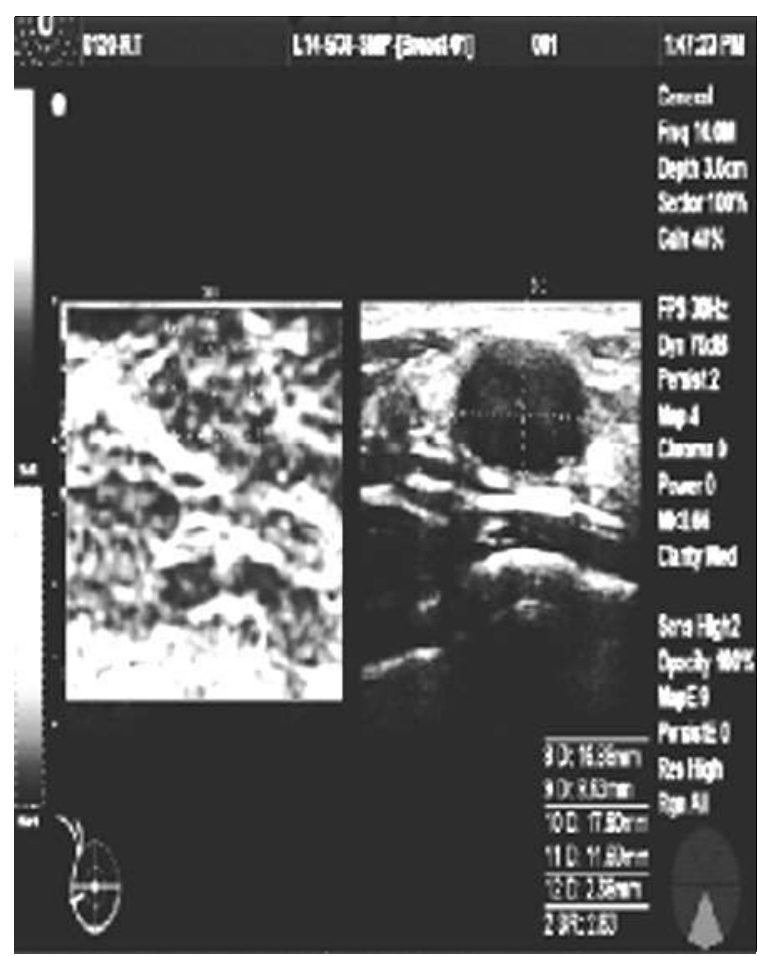

Fig. 3 Example of ultrasound image lesion scan where the specific individual region of interest (ROI) was indicated by a radiologist.
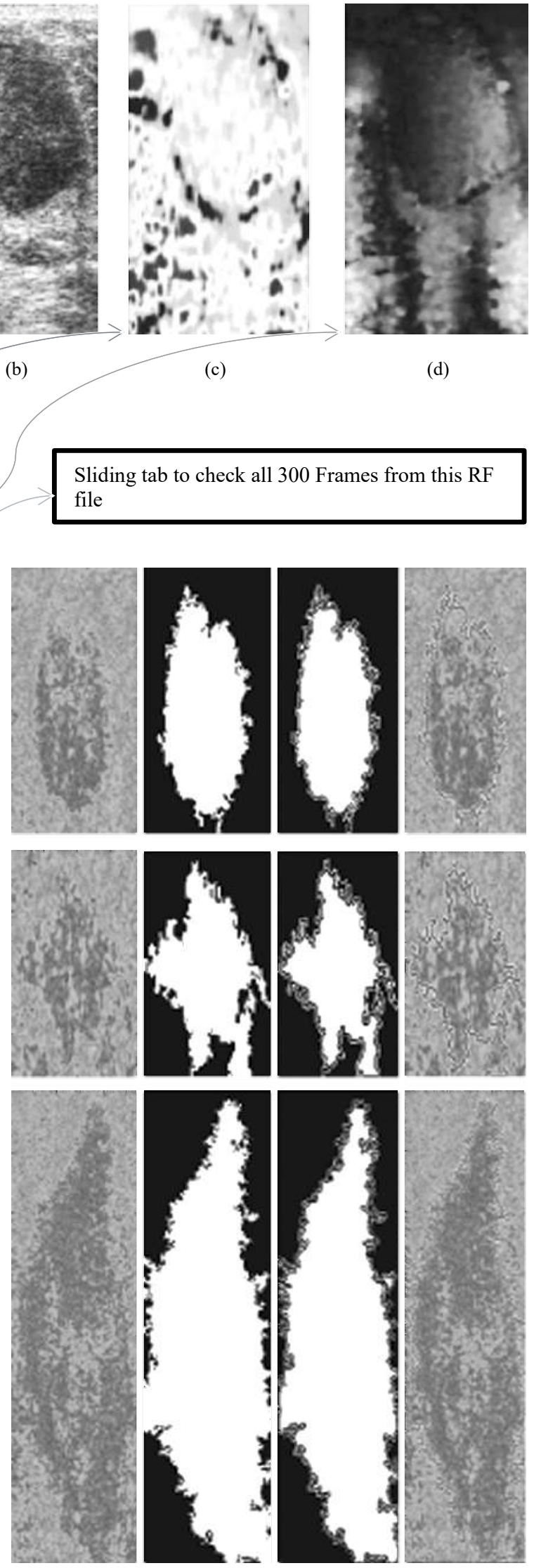

Fig. 4 Simulation results of auto segmentation on breast masses. The first two rows presented benign masses and the third row depicted malignant masses. Each row illustrated the B-Mode images in the first column, corresponding binary images in the second column, simulation results of auto segmentation on binary and B-Mode images are on the third and fourth columns respectively. 
13 benign lesions did not qualify for a biopsy. Instead, they were observed by the radiologist over a two-year period. Experimental results of auto lesion detection are presented in Fig. 4 where it is shown that the lesion boundary regions are detected precisely. Two benign and one malignant masses' detection results are shown in Fig. 4. In auto lesion boundary detection process the region of interest (ROI) is cropped by a radiologist from the B-Mode image then this cropped B-Mode image is subjected to binarization. All the experimental results are judgment by expert radiologists for all 100 ultrasound images proving that the final boundary detected by the proposed method is highly accurate.

\section{V.CONCLUSION}

In this paper the proposed auto segmentation (i.e. lesion boundary detection) process applied on both the B-Mode and the binary images. Because, the lesion boundary coordinates measured from the binary image are utilized for auto segmentation on B-Mode ultrasound analysis. So, image binarization is executed precisely in this proposed method. Many statistical and geometrical features can be extracted from the segmented lesion contour for classification of breast tumors on B-Mode ultrasound analysis. This paper is more likely as a feasibility study of auto segmentation task. Moreover, this proposed method is observed on a limited clinical case of 100 patients. A wide range of patients should be studied to establish this method.

\section{REFERENCES}

[1] American Cancer Society website (2018), [Online]. Available: https://www.cancer.org/content/dam/cancer-org/research/ cancer-facts-and-statistics/annual-cancer-facts-and-figures/ 2018/leading-sites-of-new-cancer-cases-and-deaths-2018estimates.pdf

[2] Mohd Anisur Rahman Forazy,"Incidence of breast cancer in Bangladesh," Health Care, vol. 3, issue 3, pp. 53-54, October 2015.

[3] Steven Halls (2018), "Moose \& Doc Breast Cancer." [Online]. Available: https://breast-cancer.ca/ult-bens

[4] Karla Horsch, Maryellen L. Giger, Luz A. Venta, and Carl J. Vyborny, "Computerized diagnosis of breast lesions on ultrasound" Medical Physics, vol. 29, pp. 157-164, 2002.

[5] Wei-Chih Shen, MS, Ruey-Feng Chang, PhD, Woo Kyung Moon, MD, Yi-Hong Chou, MD, Chiun-Sheng Huang, MD, "Breast Ultrasound Computer-Aided Diagnosis Using BIRADS Features," Academic Radiology, Vol. 14, No. 8, pp. 928-939, 2007.

[6] Sharmin R. Ara, Syed Khairul Bashar, Farzana Alam, Md. Kamrul Hasan, "EMD-DWT Based Transform Domain Feature Reduction Approach for Quantitative Multi-class Classification of Breast Tumors", Ultrasonics, Vol. 80, pp. 22 33, 2017.

[7] Wikipedia (2018), The Free Encyclopedia website. [Online]. Available: https://en.wikipedia.org/wiki/Medical_ultrasound\# Modes

[8] Wikipedia (2018), The Free Encyclopedia website. [Online]. Available: https://en.wikipedia.org/wiki/Binary_image

[9] Hanna Piotrzkowska-Wróblewska, Katarzyna DobruchSobczak, Michał Byra and Andrzej Nowicki "Open access database of raw ultrasonic signals acquired from malignant and benign breast lesions," Department of Ultrasound, Institute of Fundamental Technological Research, Polish Academy of Sciences, Poland, Medical Physics Dataset Article, 2017.

[10] Thomas Kristoffersen Børstad, Comparison of three ultrasound velocity estimators for strain imaging of the brain,
Norwegian University of Science and Technology, NTNU 100 skapende $\dot{a}$ r press, Trondheim, Norway, December 2010.

[11] Thomas Kristoffersen Børstad, "Intraoperative Ultrasound Strain Imaging of Brain Tumours," M.Sc. Eng. thesis, Norwegian University of Science and Technology, Norway, June 2011.

[12] Gonzalez, R. C., R. E. Woods, and S. L. Eddins, Digital Image Processing Using MATLAB, Gatesmark Publishing, 2009.

[13] Shahriar Mahmud Kabir and Mohammed Imamul Hassan Bhuiyan, "Classification of Breast Tumour in Contourlet Transform Domain" Proceedings of the 10th International Conference on Electrical and Computer Engineering (ICECE), pp. 289-292, 2018.

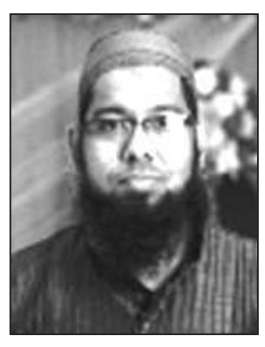

Shahriar Mahmud Kabir received the B.Sc. in Electrical and Electronic Engineering (EEE) from Ahsanullah University of Science and Technology (AUST) at 2008 and M.Sc. in Electrical and Electronic Engineering (EEE) from Bangladesh University of Engineering and Technology (BUET) at 2013. Currently, he is pursuing his Ph.D. degree in Electrical and Electronic Engineering (EEE) at Bangladesh University of Engineering and Technology (BUET). He can be reached at Green University of Bangladesh (GUB), Assistant Professor, Department of Electrical and Electronic Engineering (EEE), Dhaka-1207. Email: kabir@eee.green.edu.bd

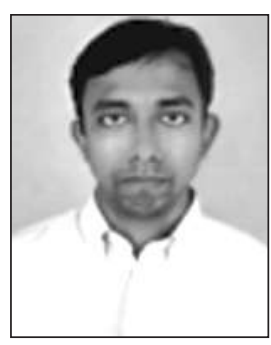

Mohammed Imamul Hassan Bhuiyan received the B.Sc. and M.Sc. in EEE from Bangladesh University of Engineering and Technology (BUET) in 1998 and 2001 respectively. He did his Ph.D. degree in Electrical and Computer Engineering from Concordia University, Montreal, Canada, in 2007. Currently, he is the honorable Chairperson in the Department of Biomedical Engineering in BUET. He can be reached at imamul@eee.buet.ac.bd 